Article

\title{
Simple Sufficient Subordination Conditions for Close-to-Convexity
}

\author{
Ebrahim Analouei Adegani ${ }^{1}$, Teodor Bulboacă ${ }^{2, *}$ (I) and Ahmad Motamednezhad ${ }^{1}$
}

1 Faculty of Mathematical Sciences, Shahrood University of Technology, P.O. Box 316-36155, Shahrood, Iran; analoey.ebrahim@gmail.com (E.A.A.); a.motamedne@gmail.com (A.M.)

2 Faculty of Mathematics and Computer Science, Babeş-Bolyai University, 400084 Cluj-Napoca, Romania

* Correspondence: bulboaca@math.ubbcluj.ro; Tel.: +40-729-087-153

Received: 29 January 2019; Accepted: 6 March 2019; Published: 7 March 2019

Abstract: Using several applications of the theory of differential subordination we obtain sufficient conditions for usually normalized analytic functions to belong to certain subclasses of close-to-convex functions and close-to-convex functions of order $\alpha$.

Keywords: analytic functions; univalent function; differential subordination; starlike and close-to-convex functions of order $\alpha$

MSC: primary 30C45; secondary 30C 80

\section{Introduction and Preliminaries}

Let $\mathcal{A}$ be the class of functions with power series expansions

$$
f(z)=z+\sum_{n=2}^{\infty} a_{n} z^{n}
$$

that are analytic in the open unit disk $\mathbb{U}:=\{z \in \mathbb{C}:|z|<1\}$, and denote by $\mathcal{S}$ the class of all functions of $\mathcal{A}$ which are univalent in $\mathbb{U}$.

For the functions $f$ and $F$ which are analytic in $\mathbb{U}$, the function $f$ is said to be subordinate to $F$, and write $f(z) \prec F(z)$, if there exists a Schwarz function $\omega$, which is analytic in $\mathbb{U}$ with $\omega(0)=0$ and $|\omega(z)|<1$, such that $f(z)=F(\omega(z))$ for all $z \in \mathbb{U}$.

By Schwarz lemma we have $|\omega(z)| \leq|z|, z \in \mathbb{U}$, which concludes that $\omega(\mathbb{U}) \subset \mathbb{U}$. Since $\omega(0)=0$ and $\omega(\mathbb{U}) \subset \mathbb{U}$ it follows that if $f(z) \prec F(z)$, then $f(0)=F(0)$ and $f(\mathbb{U}) \subset F(\mathbb{U})$. In particular, if the function $F$ is univalent in $\mathbb{U}$, then we have the following equivalence

$$
f(z) \prec F(z) \Leftrightarrow f(0)=F(0) \quad \text { and } \quad f(\mathbb{U}) \subset F(\mathbb{U}) .
$$

We denote by $\mathcal{S}^{*}(\alpha)$ the subclass of $\mathcal{A}$ consisting of functions which are starlike of order $\alpha$, as follows:

$$
\mathcal{S}^{*}(\alpha):=\left\{f \in \mathcal{A}: \operatorname{Re} \frac{z f^{\prime}(z)}{f(z)}>\alpha, z \in \mathbb{U}\right\}, 0 \leq \alpha<1,
$$

and, in particular, $\mathcal{S}^{*}:=\mathcal{S}^{*}(0)$ is the class of starlike functions in the unit disk $\mathbb{U}$.

Also, we denote by $\mathcal{C}(\alpha)$ the subclass of $\mathcal{A}$ consisting of functions which are close-to-convex of order $\alpha$ if there exists a function $g \in \mathcal{S}^{*}$ such that

$$
\operatorname{Re} \frac{z f^{\prime}(z)}{g(z)}>\alpha, z \in \mathbb{U}, 0 \leq \alpha<1 .
$$


In particular, $\mathcal{C}:=\mathcal{C}(0)$ is the class of close-to-convex functions in the unit disk $\mathbb{U}$. It is well known that $\mathcal{S}^{*}(\alpha) \subset \mathcal{S}$ and $\mathcal{C}(\alpha) \subset \mathcal{S}$, for all $0 \leq \alpha<1$.

The idea of subordination was used for defining many of classes of functions studied in the Geometric Function Theory. Nunokawa et al. [1] showed that if $p$ is an analytic function in $\mathbb{U}$ with $p(0)=1$, then

$$
1+z p^{\prime}(z) \prec 1+z \Rightarrow p(z) \prec 1+z .
$$

They applied this differential implication to obtain a criterion for normalized analytic functions to be univalent. In the literature, several authors obtained several applications in the geometric functions theory by using differential subordination, for example see [2-8].

The following lemmas will be used in our investigation:

Lemma 1. ([9], Theorem $3.4 \mathrm{~h}, \mathrm{p} .132)$ Let $q$ be analytic in $\mathbb{U}$ and let $\psi$ and $\theta$ be analytic in a domain $D$ containing $q(\mathbb{U})$ with $\psi(w) \neq 0$ when $w \in q(\mathbb{U})$. Set $Q(z):=z q^{\prime}(z) \psi(q(z))$ and $h(z):=\theta(q(z))+Q(z)$. Suppose that:

(i) either $h$ is convex, or $Q$ is starlike univalent in $\mathbb{U}$, and

(ii) $\operatorname{Re} \frac{z h^{\prime}(z)}{Q(z)}>0$ for $z \in \mathbb{U}$.

If $p$ is analytic in $\mathbb{U}$, with $p(0)=q(0), p(\mathbb{U}) \subseteq D$ and

$$
\theta(p(z))+z p^{\prime}(z) \psi(p(z)) \prec \theta(q(z))+z q^{\prime}(z) \psi(q(z)),
$$

then $p(z) \prec q(z)$, and $q$ is best dominant of $(1)$.

Lemma 2. $[10,11]$ Let $p(z)=1+\sum_{n \geq m}^{\infty} c_{n} z^{n}, c_{m} \neq 0$, be an analytic function in $|z|<1$ with $p(0)=1$ and $p(z) \neq 0$ for $z \in \mathbb{U}$. If there exists a point $z_{0}$ with $\left|z_{0}\right|<1$, such that

$$
|\arg p(z)|<\frac{\beta \pi}{2}, \quad \text { for } \quad|z|<\left|z_{0}\right|,
$$

and

$$
\left|\arg p\left(z_{0}\right)\right|=\frac{\beta \pi}{2}
$$

for some $\beta>0$, then we have

$$
\frac{z_{0} p^{\prime}\left(z_{0}\right)}{p\left(z_{0}\right)}=\frac{2 i k \arg p\left(z_{0}\right)}{\pi}
$$

for some $k \geq \frac{m\left(a+a^{-1}\right)}{2} \geq m$, where

$$
\left[p\left(z_{0}\right)\right]^{1 / \beta}= \pm i a, \quad \text { and } a>0 .
$$

In this article we will show several applications of the theory of differential subordination to obtain simple sufficient conditions for normalized analytic functions to belong to certain subclasses of close-to-convex functions of order $\alpha$.

\section{Sufficient Conditions for Close-to-Convexity and Applications}

Theorem 1. Let the function $q$ be univalent in $\mathbb{U}$ such that $q(0)=1$ and satisfies

$$
\operatorname{Re}\left[1+\frac{z q^{\prime \prime}(z)}{q^{\prime}(z)}\right]>\max \left\{0 ;-\operatorname{Re} \frac{1}{\lambda}\right\}, z \in \mathbb{U},
$$


where $\lambda \in \mathbb{C}$, with $|\lambda|^{2}+\operatorname{Re} \lambda>0$. If $f \in \mathcal{A}$ and $g \in \mathcal{S}^{*}$ such that

$$
\frac{\lambda z f^{\prime}(z)}{g(z)}\left(1+\frac{1}{\lambda}+\frac{z f^{\prime \prime}(z)}{f^{\prime}(z)}-\frac{z g^{\prime}(z)}{g(z)}\right) \prec q(z)+\lambda z q^{\prime}(z),
$$

then

$$
\frac{z f^{\prime}(z)}{g(z)} \prec q(z)
$$

and $q$ is the best dominant of (3).

Proof. Since $\lambda \in \mathbb{C}$ with $|\lambda|^{2}+\operatorname{Re} \lambda>0$, then

$$
\max \left\{0 ;-\operatorname{Re} \frac{1}{\lambda}\right\}<1
$$

For $f \in \mathcal{A}$ and $g \in \mathcal{S}^{*}$, let

$$
p(z):=\frac{z f^{\prime}(z)}{g(z)}, z \in \mathbb{U} .
$$

Since all starlike functions are univalent, it follows that $g(z) \neq 0$ for all $z \in \mathbb{U} \backslash\{0\}$, and $z_{0}=0$ is a simple zero for $g$, it follows that $p$ is analytic in $\mathbb{U}$. Moreover, using the fact that $f, g \in \mathcal{A}$, then $f$ and $g$ are of the form $f(z)=z+\sum_{n=2}^{\infty} a_{n} z^{n}$ and $g(z)=z+\sum_{n=2}^{\infty} b_{n} z^{n}$, hence

$$
p(z):=\frac{z f^{\prime}(z)}{g(z)}=\frac{1+\sum_{n=2}^{\infty} n a_{n} z^{n-1}}{1+\sum_{n=2}^{\infty} n b_{n} z^{n-1}}, z \in \mathbb{U},
$$

and therefore, $p(0)=1$. A simple computation shows that

$$
p(z)+\lambda z p^{\prime}(z)=\frac{\lambda z f^{\prime}(z)}{g(z)}\left(1+\frac{1}{\lambda}+\frac{z f^{\prime \prime}(z)}{f^{\prime}(z)}-\frac{z g^{\prime}(z)}{g(z)}\right), z \in \mathbb{U} .
$$

To prove our result by using Lemma 1 , we define the functions $\theta(w)=w$ and $\psi(w)=\lambda, w \in \mathbb{C}$. These functions are analytic in the domain $D:=\mathbb{C}$ containing $q(\mathbb{U})$ and $\psi(w) \neq 0$ for all $w \in q(\mathbb{U})$. Let $Q, h: \mathbb{U} \rightarrow \mathbb{C}$ be defined by

$$
Q(z):=z q^{\prime}(z) \psi(q(z))=\lambda z q^{\prime}(z)
$$

and

$$
h(z):=\theta(q(z))+Q(z)=q(z)+\lambda z q^{\prime}(z) .
$$

Since $Q(0)=0$ and $Q^{\prime}(0)=\lambda q^{\prime}(0) \neq 0$, assumption (2) shows that

$$
\operatorname{Re} \frac{z Q^{\prime}(z)}{Q(z)}=\operatorname{Re}\left[1+\frac{z q^{\prime \prime}(z)}{q^{\prime}(z)}\right]>0, z \in \mathbb{U},
$$

and therefore $Q$ is a starlike (univalent) function in $\mathbb{U}$. On other hand

$$
\operatorname{Re} \frac{z h^{\prime}(z)}{Q(z)}=\operatorname{Re}\left[1+\frac{1}{\lambda}+\frac{z q^{\prime \prime}(z)}{q^{\prime}(z)}\right]>0, z \in \mathbb{U},
$$

and

$$
\frac{\lambda z f^{\prime}(z)}{g(z)}\left(1+\frac{1}{\lambda}+\frac{z f^{\prime \prime}(z)}{f^{\prime}(z)}-\frac{z g^{\prime}(z)}{g(z)}\right)=\theta(p(z))+z p^{\prime}(z) \psi(p(z)) \prec q(z)+\lambda z q^{\prime}(z)
$$


Therefore, all the conditions of Lemma 1 are satisfied, which implies $p(z) \prec q(z)$, and the function $q$ is the best dominant of (3).

Remark 1. K. Sakaguchi introduced and studied in [12] the class of starlike functions with respect to symmetrical points, defined by

$$
\mathcal{S}^{s}:=\left\{f \in \mathcal{A}: \operatorname{Re} \frac{z f^{\prime}(z)}{f(z)-f(-z)}>0, z \in \mathbb{U}\right\} .
$$

From the proof of Theorem 1 of [12] it follows that

$$
f \in \mathcal{S}^{s} \Rightarrow g(z)=: \frac{f(z)-f(-z)}{2} \in \mathcal{S}^{*},
$$

and therefore $\mathcal{S}^{s} \subset \mathcal{C}$.

Consequently, from the above theorem we deduce the next result: assuming that $f \in \mathcal{S}^{s}$ and (3) holds for $g(z)=: \frac{f(z)-f(-z)}{2}$, then $\frac{z f^{\prime}(z)}{g(z)} \prec q(z)$ and $q$ is the best dominant of (3). We mention that this result could be connected with those obtained by Bukhari et al. ([2], Theorem 1).

Corollary 1. For $-1 \leq B<A \leq 1$, if $f \in \mathcal{A}$ and $g \in \mathcal{S}^{*}$ such that

$$
\frac{z f^{\prime}(z)}{g(z)}\left(2+\frac{z f^{\prime \prime}(z)}{f^{\prime}(z)}-\frac{z g^{\prime}(z)}{g(z)}\right) \prec \frac{1+A z}{1+B z}+\frac{(A-B) z}{(1+B z)^{2}}
$$

then

$$
\frac{z f^{\prime}(z)}{g(z)} \prec \frac{1+A z}{1+B z}
$$

that is $f \in \mathcal{C}\left(\frac{1-A}{1-B}\right)$, and $q(z)=\frac{1+A z}{1+B z}$ is the best dominant of (4).

Proof. For $q(z)=\frac{1+A z}{1+B z}$, where $-1 \leq B<A \leq 1$, and $\lambda=1$ it is easy to check that the inequality (2) holds, and from Theorem 1 we obtain that $\frac{z f^{\prime}(z)}{g(z)} \prec \frac{1+A z}{1+B z}$, and $q(z)=\frac{1+A z}{1+B z}$ is the best dominant of (4). Since the function $q$ is a circular transform, it is easy to check that

$$
q(\mathbb{U})=\left\{w \in \mathbb{C}:\left|w-\frac{1-A B}{1-B^{2}}\right|<\frac{A-B}{1-B^{2}}\right\}, \text { if }-1<B<A \leq 1,
$$

and

$$
q(\mathbb{U})=\left\{w \in \mathbb{C}: \operatorname{Re} w>\frac{1-A}{2}\right\}, \text { if } B=-1,
$$

hence the above subordination implies $f \in \mathcal{C}\left(\frac{1-A}{1-B}\right)$.

For $A=1-2 \alpha$ and $B=-1$, where $0 \leq \alpha<1$, Corollary 1 reduces to the following example which gives sufficient condition for functions to be close-to-convex of order $\alpha$ :

Example 1. For $0 \leq \alpha<1$, if $f \in \mathcal{A}$ and $g \in \mathcal{S}^{*}$ such that

$$
\frac{z f^{\prime}(z)}{g(z)}\left(2+\frac{z f^{\prime \prime}(z)}{f^{\prime}(z)}-\frac{z g^{\prime}(z)}{g(z)}\right) \prec \frac{1+(1-2 \alpha) z}{1-z}+\frac{2(1-\alpha) z}{(1-z)^{2}}
$$


then

$$
\frac{z f^{\prime}(z)}{g(z)} \prec \frac{1+(1-2 \alpha) z}{1-z},
$$

that is $f \in \mathcal{C}(\alpha)$, and $q(z)=\frac{1+(1-2 \alpha) z}{1-z}$ is the best dominant of (5).

Corollary 2. If $f \in \mathcal{A}$ and $g \in \mathcal{S}^{*}$ such that

$$
\frac{z f^{\prime}(z)}{g(z)}\left(2+\frac{z f^{\prime \prime}(z)}{f^{\prime}(z)}-\frac{z g^{\prime}(z)}{g(z)}\right) \prec e^{z}(1+z),
$$

then

$$
\frac{z f^{\prime}(z)}{g(z)} \prec e^{z}
$$

and $q(z)=e^{z}$ is the best dominant of (6).

Proof. For $q(z)=e^{z}$ and $\lambda=1$ it is easy to check that the inequality (2), and from Theorem 1 we obtain our result.

Theorem 2. Let the function $q$ be univalent in $\mathbb{U}$ such that $q(0)=1$ and satisfies

$$
\operatorname{Re}\left[1+\frac{z q^{\prime \prime}(z)}{q^{\prime}(z)}-\frac{z q^{\prime}(z)}{q(z)}\right]>0, z \in \mathbb{U},
$$

and

$$
\operatorname{Re}\left[1+q(z)+\frac{z q^{\prime \prime}(z)}{q^{\prime}(z)}-\frac{z q^{\prime}(z)}{q(z)}\right]>0, z \in \mathbb{U} .
$$

If $f \in \mathcal{A}$ and $g \in \mathcal{S}^{*}$ such that

$$
1+\frac{z f^{\prime \prime}(z)}{f^{\prime}(z)}+\frac{z f^{\prime}(z)}{g(z)}-\frac{z g^{\prime}(z)}{g(z)} \prec q(z)+\frac{z q^{\prime}(z)}{q(z)}
$$

then

$$
\frac{z f^{\prime}(z)}{g(z)} \prec q(z)
$$

and $q$ is the best dominant of (9).

Proof. For $f \in \mathcal{A}$ and $g \in \mathcal{S}^{*}$, if we set

$$
p(z):=\frac{z f^{\prime}(z)}{g(z)}, z \in \mathbb{U}
$$

then $p$ is analytic in $\mathbb{U}$, with $p(0)=1$, and we could easily check that

$$
p(z)+\frac{z p^{\prime}(z)}{p(z)}=1+\frac{z f^{\prime \prime}(z)}{f^{\prime}(z)}+\frac{z f^{\prime}(z)}{g(z)}-\frac{z g^{\prime}(z)}{g(z)}, z \in \mathbb{U} .
$$

First, we will prove that $0 \notin q(\mathbb{U})$. Otherwise, if there exists $z_{0} \in \mathbb{U}$ such that $q\left(z_{0}\right)=0$, from the univalence of $q$ it follows that $q^{\prime}\left(z_{0}\right) \neq 0$, and from $q(0)=1$ we get that $z_{0} \in \mathbb{U} \backslash\{0\}$. Thus, the function

$$
H(z):=1+\frac{z q^{\prime \prime}(z)}{q^{\prime}(z)}-\frac{z q^{\prime}(z)}{q(z)}, z \in \mathbb{U}
$$

has a simple pole at the point $z_{0} \in \mathbb{U} \backslash\{0\}$, which contradicts (7), then we conclude that $0 \notin q(\mathbb{U})$. 
To prove our result by using Lemma 1 we define the functions $\theta(w)=w$ and $\psi(w)=\frac{1}{w}$, $w \in \mathbb{C}^{*}:=\mathbb{C} \backslash\{0\}$. The functions $\theta$ and $\psi$ are analytic in the domain $D:=\mathbb{C}^{*}$ containing $q(\mathbb{U})$, and $\psi(w) \neq 0$ for $w \in q(\mathbb{U})$. If we define the functions $Q, h: \mathbb{U} \rightarrow \mathbb{C}$ by

$$
Q(z):=z q^{\prime}(z) \psi(q(z))=\frac{z q^{\prime}(z)}{q(z)}
$$

and

$$
h(z):=\theta(q(z))+Q(z)=q(z)+\frac{z q^{\prime}(z)}{q(z)}
$$

then $Q(0)=0$ and $Q^{\prime}(0)=\frac{q^{\prime}(0)}{q(0)} \neq 0$. Further computations combined with the assumption (7) show that

$$
\operatorname{Re} \frac{z Q^{\prime}(z)}{Q(z)}=\operatorname{Re}\left[1+\frac{z q^{\prime \prime}(z)}{q^{\prime}(z)}-\frac{z q^{\prime}(z)}{q(z)}\right]>0, z \in \mathbb{U},
$$

and therefore $Q$ is starlike (univalent) in $\mathbb{U}$. Moreover, the assumption (8) implies that

$$
\operatorname{Re} \frac{z h^{\prime}(z)}{Q(z)}=\operatorname{Re}\left[1+q(z)+\frac{z q^{\prime \prime}(z)}{q^{\prime}(z)}-\frac{z q^{\prime}(z)}{q(z)}\right]>0, z \in \mathbb{U}
$$

Since

$$
1+\frac{z f^{\prime \prime}(z)}{f^{\prime}(z)}+\frac{z f^{\prime}(z)}{g(z)}-\frac{z g^{\prime}(z)}{g(z)}=\theta(p(z))+z p^{\prime}(z) \psi(p(z)) \prec q(z)+\frac{z q^{\prime}(z)}{q(z)}
$$

and all conditions of Lemma 1 hold, we conclude that $p(z) \prec q(z)$, and the function $q$ is the best dominant of (9).

Corollary 3. For $-1 \leq B<A \leq 1$, if $f \in \mathcal{A}$ and $g \in \mathcal{S}^{*}$ such that

$$
1+\frac{z f^{\prime \prime}(z)}{f^{\prime}(z)}+\frac{z f^{\prime}(z)}{g(z)}-\frac{z g^{\prime}(z)}{g(z)} \prec \frac{1+A z}{1+B z}+\frac{(A-B) z}{(1+A z)(1+B z)},
$$

then

$$
\frac{z f^{\prime}(z)}{g(z)} \prec \frac{1+A z}{1+B z}
$$

that is $f \in \mathcal{C}\left(\frac{1-A}{1-B}\right)$, and $q(z)=\frac{1+A z}{1+B z}$ is the best dominant of (10).

Proof. For $q(z)=\frac{1+A z}{1+B z}$, where $-1 \leq B<A \leq 1$, it is easy to check that the inequalities (7) and (8) hold, and from Theorem 2 we get our result.

For $A=1-2 \alpha$ and $B=-1$, where $0 \leq \alpha<1$, the above corollary reduces to the following example which gives a sufficient condition for functions to be close-to-convex of order $\alpha$ :

Example 2. For $0 \leq \alpha<1$, if $f \in \mathcal{A}$ and $g \in \mathcal{S}^{*}$ such that

$$
1+\frac{z f^{\prime \prime}(z)}{f^{\prime}(z)}+\frac{z f^{\prime}(z)}{g(z)}-\frac{z g^{\prime}(z)}{g(z)} \prec \frac{1+(1-2 \alpha) z}{1-z}+\frac{2(1-\alpha) z}{[1+(1-2 \alpha) z](1-z)},
$$

then

$$
\frac{z f^{\prime}(z)}{g(z)} \prec \frac{1+(1-2 \alpha) z}{1-z}
$$


that is $f \in \mathcal{C}(\alpha)$, and $q(z)=\frac{1+(1-2 \alpha) z}{1-z}$ is the best dominant of (11).

Corollary 4. If $f \in \mathcal{A}$ and $g \in \mathcal{S}^{*}$ such that

$$
1+\frac{z f^{\prime \prime}(z)}{f^{\prime}(z)}+\frac{z f^{\prime}(z)}{g(z)}-\frac{z g^{\prime}(z)}{g(z)} \prec e^{z}+z
$$

then

$$
\frac{z f^{\prime}(z)}{g(z)} \prec e^{z}
$$

and $q(z)=e^{z}$ is the best dominant of (12).

Proof. For $q(z)=e^{z}$ it is easy to check that the inequalities (7) and (8) hold, and from Theorem 2 we get the above implication.

Theorem 3. For $0 \leq \alpha<1$, if $f \in \mathcal{A}$ and $g \in \mathcal{S}^{*}$ such that

$$
\operatorname{Re}\left[\frac{1}{1-\alpha}\left(\frac{z f^{\prime}(z)}{g(z)}-\alpha\right)+\frac{\frac{z f^{\prime}(z)}{g(z)}\left(1+\frac{z f^{\prime \prime}(z)}{f^{\prime}(z)}-\frac{z g^{\prime}(z)}{g(z)}\right)}{\frac{z f^{\prime}(z)}{g(z)}-\alpha}\right]^{2}>-1, z \in \mathbb{U},
$$

then

$$
\operatorname{Re} \frac{z f^{\prime}(z)}{g(z)}>\alpha, z \in \mathbb{U}
$$

that is $f \in \mathcal{C}(\alpha)$.

Proof. For $f \in \mathcal{A}$ and $g \in \mathcal{S}^{*}$, if we set

$$
p(z):=\frac{1}{1-\alpha}\left(\frac{z f^{\prime}(z)}{g(z)}-\alpha\right), z \in \mathbb{U}
$$

then $p$ is analytic in $\mathbb{U}$, with $p(0)=1$, and we easily deduce that

$$
p(z)+\frac{z p^{\prime}(z)}{p(z)}=\frac{1}{1-\alpha}\left(\frac{z f^{\prime}(z)}{g(z)}-\alpha\right)+\frac{\frac{z f^{\prime}(z)}{g(z)}\left(+\frac{z f^{\prime \prime}(z)}{f^{\prime}(z)}-\frac{z g^{\prime}(z)}{g(z)}\right)}{\frac{z f^{\prime}(z)}{g(z)}-\alpha}, z \in \mathbb{U} .
$$

Supposing that there exists a point $z_{0} \in \mathbb{U}$ such that

$$
\operatorname{Re} p(z)>0, \text { for }|z|<\left|z_{0}\right|,
$$

and

$$
\operatorname{Re} p\left(z_{0}\right)=0
$$

using Lemma 2 for $\beta=1$ we have

$$
\frac{z_{0} p^{\prime}\left(z_{0}\right)}{p\left(z_{0}\right)}=\frac{2 i k \arg p\left(z_{0}\right)}{\pi}
$$

for some $k \geq \frac{a+a^{-1}}{2} \geq 1$, where

$$
p\left(z_{0}\right)= \pm i a, \quad a>0 .
$$


Therefore,

$$
\operatorname{Re}\left[p\left(z_{0}\right)+\frac{z_{0} p^{\prime}\left(z_{0}\right)}{p\left(z_{0}\right)}\right]^{2}=\operatorname{Re}( \pm i a \pm i k)^{2}=-(a+k)^{2}<-k^{2} \leq-1,
$$

which, according to (14), is a contradiction with the assumption (13). It follows that $\operatorname{Re} p(z)>0$ for all $z \in \mathbb{U}$, or equivalently $\operatorname{Re} \frac{z f^{\prime}(z)}{g(z)}>\alpha, z \in \mathbb{U}$, that is $f \in \mathcal{C}(\alpha)$.

For $\alpha=0$ the above result leads to the following result which gives a sufficient close-to-convexity condition:

Corollary 5. If $f \in \mathcal{A}$ and $g \in \mathcal{S}^{*}$ such that

$$
\operatorname{Re}\left[1+\frac{z f^{\prime \prime}(z)}{f^{\prime}(z)}+\frac{z f^{\prime}(z)}{g(z)}-\frac{z g^{\prime}(z)}{g(z)}\right]^{2}>-1, z \in \mathbb{U},
$$

then

$$
\operatorname{Re} \frac{z f^{\prime}(z)}{g(z)}>0, z \in \mathbb{U}
$$

that is $f \in \mathcal{C}$.

Since the Koebe function $g_{\theta}(z)=\frac{z}{\left(1-e^{i \theta} z\right)^{2}}$, with $\theta \in \mathbb{R}$, belongs to $\mathcal{S}^{*}$ and $g(z)=\frac{z}{1-\mu z^{\prime}}$, where $\mu \in \mathbb{C}$ with $|\mu| \leq 1$, belongs to $\mathcal{S}^{*}\left(\frac{1}{1+|\mu|}\right) \subset \mathcal{S}^{*}$, the Corollary 5 leads to the following results, respectively:

Example 3. If $f \in \mathcal{A}$ and $\theta \in \mathbb{R}$ such that

$$
\operatorname{Re}\left[\left(1-e^{i \theta} z\right)^{2} f^{\prime}(z)+\frac{z f^{\prime \prime}(z)}{f^{\prime}(z)}-\frac{2 e^{i \theta} z}{1-e^{i \theta} z}\right]^{2}>-1, z \in \mathbb{U},
$$

then

$$
\operatorname{Re}\left(1-e^{i \theta} z\right)^{2} f^{\prime}(z)>0, z \in \mathbb{U}
$$

that is $f \in \mathcal{C}$.

Example 4. If $f \in \mathcal{A}$ and $\mu \in \mathbb{C}$, with $|\mu| \leq 1$, such that

$$
\operatorname{Re}\left[(1-\mu z) f^{\prime}(z)+\frac{z f^{\prime \prime}(z)}{f^{\prime}(z)}-\frac{\mu z}{1-\mu z}\right]^{2}>-1, z \in \mathbb{U},
$$

then

$$
\operatorname{Re}(1-\mu z) f^{\prime}(z)>0, z \in \mathbb{U}
$$

that is $f \in \mathcal{C}$.

From the above two examples we remark that the inequalities (15) and (16) represent sufficient conditions for a function $f \in \mathcal{A}$ to belong to the classes

$$
\mathcal{K}_{\theta}:=\left\{f \in \mathcal{A}: \operatorname{Re}\left(1-e^{i \theta} z\right)^{2} f^{\prime}(z)>0, z \in \mathbb{U}\right\}, \theta \in \mathbb{R}
$$


and

$$
\mathcal{F}_{\mu}:=\left\{f \in \mathcal{A}: \operatorname{Re}(1-\mu z) f^{\prime}(z)>0, z \in \mathbb{U}\right\}, \mu \in \mathbb{C},|\mu| \leq 1,
$$

respectively, that are well known subclasses of $\mathcal{C}$.

Concluding, all the three theorems of our paper give some simple conditions for close-to-convexity, and are followed by useful applications where the dominants are circular transforms and exponential functions. The results of these corollaries and examples are not trivial and could not be easily obtained by using direct computations, but there are immediate consequences of the main theorems of this paper. We are sure that our main results could be easily and successfully used to prove the close-to-convexity of other type of functions by choosing appropriate dominants.

Author Contributions: The authors contributed equally to this work.

Funding: This research received no external funding.

Acknowledgments: The authors are grateful to the reviewers of this article, that gave valuable remarks, comments, and advices, in order to revise and improve the results of the paper.

Conflicts of Interest: The authors declare no conflict of interest.

\section{References}

1. Nunokawa, M.; Obradović, M.; Owa, S. One criterion for univalency. Proc. Am. Math. Soc. 1989, 106, 1035-1037. [CrossRef]

2. Bukhari, S.Z.H.; Bulboacă, T.; Shabbir, M.S. Subordination and superordination results for analytic functions with respect to symmetrical points. Quaest. Math. 2018, 41, 65-79. [CrossRef]

3. Bulboacă, T. On some classes of differential subordinations. Stud. Univ. Babeş-Bolyai Math. 1986, 31, 45-50.

4. Bulboacă, T. On some classes of differential subordinations (II). Stud. Univ. Babeş-Bolyai Math. 1986, 31, 13-21.

5. $\quad$ El-Ashwah, R.M.; Aouf, M.K.; Bulboacă, T. Differential subordinations for classes of meromorphic p-valent functions defined by multiplier transformations. Bull. Aust. Math. Soc. 2011, 83, 353-368. [CrossRef]

6. Kanas, S.; Tudor, A.E. Differential subordinations and harmonic means. Bull. Malays. Math. Sci. Soc. 2015, 38, 1243-1253. [CrossRef]

7. Kumar, S.; Ravichandran, V. Subordinations for functions with positive real part. Complex Anal. Oper. Theory 2018, 12, 1179-1191. [CrossRef]

8. Nunokawa, M.; Sokół, J. On some differential subordinations. Stud. Sci. Math. Hungar. 2017, 54, 436-445. [CrossRef]

9. Miller, S.S.; Mocanu, P.T. Differential Subordinations. Theory and Applications; Marcel Dekker Inc.: New York, NY, USA, 2000; ISBN 0-8247-0029-5.

10. Nunokawa, M. On properties of non-Carathéodory functions. Proc. Jpn. Acad. Ser. A 1992, 68, 152-153. [CrossRef]

11. Nunokawa, M. On the order of strongly starlikeness of strongly convex functions. Proc. Jpn. Acad. Ser. A Math. Sci. 1993, 69, 234-237. [CrossRef]

12. Sakaguchi, K. On a certain univalent mapping. J. Math. Soc. Jpn. 1959, 11, 72-75. [CrossRef] 